\title{
Change-Point Analysis of Tropical Night Occurrences for Five Major Cities in Republic of Korea
}

\author{
Myoung-Seok Suh ${ }^{1}$ and Chansoo Kim ${ }^{2}$ \\ ${ }^{1}$ Department of Atmospheric Science, Kongju National University, Gongju 314-701, Republic of Korea \\ ${ }^{2}$ Department of Applied Mathematics, Kongju National University, Gongju 314-701, Republic of Korea \\ Correspondence should be addressed to Chansoo Kim; chanskim@kongju.ac.kr
}

Received 11 September 2014; Revised 12 January 2015; Accepted 12 January 2015

Academic Editor: Zhihua Zhang

Copyright (c) 2015 M.-S. Suh and C. Kim. This is an open access article distributed under the Creative Commons Attribution License, which permits unrestricted use, distribution, and reproduction in any medium, provided the original work is properly cited.

\begin{abstract}
Bayesian change-point analysis is applied to detect a change-point in the occurrences of tropical night (TN) days in the 50-year time series data for five major cities in Republic of Korea. A TN day is simply defined as a day when the daily minimum temperature is greater than $25^{\circ} \mathrm{C}$. A Bayesian analysis is performed for detecting a change-point at an unknown time point in the TN day frequency time series, which is modeled by an independent Poisson random variable. The results showed that a single change occurred around 1993 for three cities (Seoul, Incheon, and Daegu). However, when we excluded the extraordinary year, 1994, a single change occurred around 1993 only in Seoul and Daegu. The average number of TN days in Seoul and Daegu increased significantly, by more than $150 \%$, after the change-point year. The abrupt increase in TN day frequency in two cities over Republic of Korea around 1993 may be related to the significant decadal change in the East Asian summer monsoon around the mid 1990s and to rapid urbanization.
\end{abstract}

\section{Introduction}

Extreme weather and climate events have wide-ranging impacts on human society as well as on biophysical systems [1-3]. Owing to their potential of causing damage to life and property, extreme weather events, such as a heat wave, tropical night (TN), and extreme rainfall events, have drawn increased attention in recent decades [4-13]. In particular, as the population increases and infrastructure develops in urban areas vulnerable to weather extremes, sudden temporal changes (abrupt regime shifts) in climate time series have become increasingly important issues [14-17]. In addition, increases in global mean air temperature have been widely observed owing to the combined effects of enhanced atmospheric greenhouse gases, such as $\mathrm{CO}_{2}, \mathrm{CH}_{4}$, and $\mathrm{O}_{3}$, and the urban heat island effect [18]. In particular, given that possible increases in the frequency and intensity of extremely hot weather events arising from global warming may have a greater impact on human health and mortality than any other form of severe weather, public concern regarding such events has greatly increased $[3,19]$.
Tank and Können [20] showed that, during an episode of pronounced warming in Europe, the annual number of warm extremes increased twice faster than expected from the corresponding decrease in the number of cold extremes for the 1976-99 subperiod. Kuglitsch et al. [12] also showed that the mean heat wave intensity, heat wave length, and heat wave number across the eastern Mediterranean region have significantly increased since the 1960s. It is well known that a significant increase in the annual occurrence of warm nights or TN days arises because of a greater increase in daily minimum temperature than that in the maximum temperature, which is caused by the urban heat island effect [21-24]

Several studies based on ground observation data have examined the linear trend and/or sudden change (regime shift) in extreme weather events over Republic of Korea [25-29]. They showed that significant increases and/or sudden changes in extreme temperature and precipitation have occurred during recent decades over Republic of Korea. In particular, the temperature, including daily maximum/minimum temperature over Republic of Korea, 
significantly increased in recent years due to the combined effects of global warming and rapid urbanization [30-32]. As a result, the frequency and intensity of extreme warm (cold) temperature events, such as warm days, heat spell, TN days, and frost days, are clearly increased (decreased). Choi and Kwon [31] showed that the occurrences of TN days are more pronounced in the evening (21 LST) and during the Changma break period (late July-early August) and that TN days in the evening frequently occur in urbanized inland cities at low latitudes due to urban heat islands. In addition, they showed that occurrences of TN days in urban areas have increased since the 1990s due to the temporal changes of the late Changma period and intensified urbanization. $\mathrm{Ha}$ and Yun [24] showed that the strong upward trend of TN days in Seoul is significantly modulated by both water vapor and air temperature, especially in July and August. Although numerous studies have been performed on the trends in extreme temperature and their impacts on mortality over Republic of Korea, relatively fewer studies have been performed to investigate the existence of a change-point in extreme temperature, especially in TN days using long-term data $[32,33]$.

The goal of this study is to investigate the existence of a change-point in the time series of TN days using Bayesian change-point analysis during a 50-year period (1958-2007) over Republic of Korea. When we take into consideration the fact that TN days have an impact on human health and/or condition, it is meaningful to investigate the change-point in time series of TN days. Bayesian analysis is selected because it is known to be an efficient way to provide a coherent and rational framework for distilling uncertainties by incorporating diverse information sources such as subjective beliefs, historical observations, and numerical model simulations $[15,17,29,34,35]$.

The outline of this paper is as follows. The data used in this study and the method to obtain the TN days are presented in Section 2. Bayesian model selection and estimation for the selected model are presented in Section 3. Detailed results and discussions of the change-point of TN days over Republic of Korea are shown in Section 4. A brief summary of the results and conclusions are given in Section 5 .

\section{Data}

The lack of high-quality, long-term climate data with the time resolution appropriate for analyzing extreme temperature is one of the biggest problems in performing analyses of extreme climate events. The number of observing stations operated by the Korea Meteorological Administration has steadily increased since the early 1970s. As a result, the current number of observing stations is 80 . The observing stations used in this study were selected to obtain the longest recording period with strict criteria. Stations with missing data for more than three days per year, especially during the warm months (June, July, August, and September), or that have been observing for less than 50 years were excluded. However, we did not take into consideration the movement of the observing station and changes of the observing environment

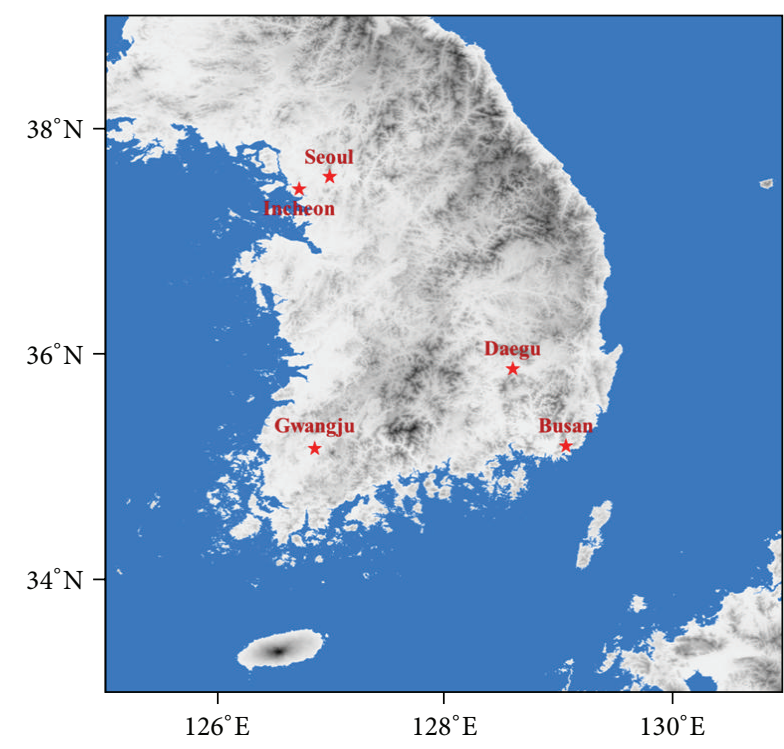

FIgURE 1: Topography and geographic locations of the five cities in Republic of Korea used in this study.

TABLE 1: Geographic characteristics of the five cities used in this study.

\begin{tabular}{lcccc}
\hline & Lat. & Lon. & Elevation $(\mathrm{m})$ & $\begin{array}{c}\text { Population } \\
\text { (March 2007) }\end{array}$ \\
\hline Seoul & $37.57 \mathrm{~N}$ & $126.98 \mathrm{E}$ & 39 & $10,200,854$ \\
Incheon & $37.46 \mathrm{~N}$ & $126.71 \mathrm{E}$ & 42 & $2,634,646$ \\
Daegu & $35.87 \mathrm{~N}$ & $128.60 \mathrm{E}$ & 49 & $2,497,019$ \\
Gwangju & $35.16 \mathrm{~N}$ & $126.85 \mathrm{E}$ & 21 & $1,410,704$ \\
Busan & $35.18 \mathrm{~N}$ & $129.08 \mathrm{E}$ & 16 & $3,607,392$ \\
\hline
\end{tabular}

and of observing instruments, because there is not enough documentation. To examine the existence of a change-point in time series of TN days, only 5 stations of daily minimum temperature among the current 80 observing stations from 1958 to 2007 over Republic of Korea met the criteria to be used in this study (Figure 1 and Table 1). Three cities (Seoul, Daegu, and Gwangju) are located in land, whereas the other two cities (Busan and Incheon) are located in coastal areas. The topography of all five cities is less than $50 \mathrm{~m}$. The population of the selected cities is very similar except for Seoul, which has more than 10 million inhabitants. Since extreme hot temperatures occurred during summer in Republic of Korea, only hot summer-season (June-September) observations are used for this study.

Although there is no uniform definition to quantify a TN day, TN days generally are defined as the occurrence of days of extreme high temperatures, typically based on a threshold value (e.g., $20^{\circ} \mathrm{C}$ or $25^{\circ} \mathrm{C}$ ) of daily minimum temperature. In this study, a TN day is defined as a day with a daily minimum temperature greater than $25^{\circ} \mathrm{C}$ as in Ha and Yun [24].

Figure 2 shows the time series of frequency of TN days in the five cities considered in Republic Korea from 1958 to 2007. The number of TN days has clearly increased in all the five cities over recent decades, especially in Seoul and Daegu. The 


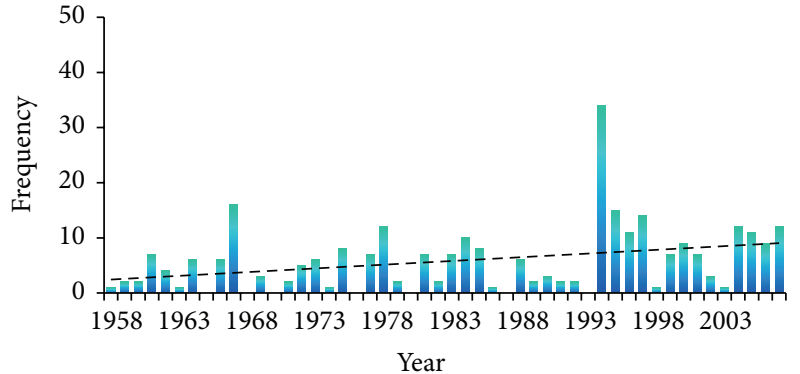

(a) Seoul

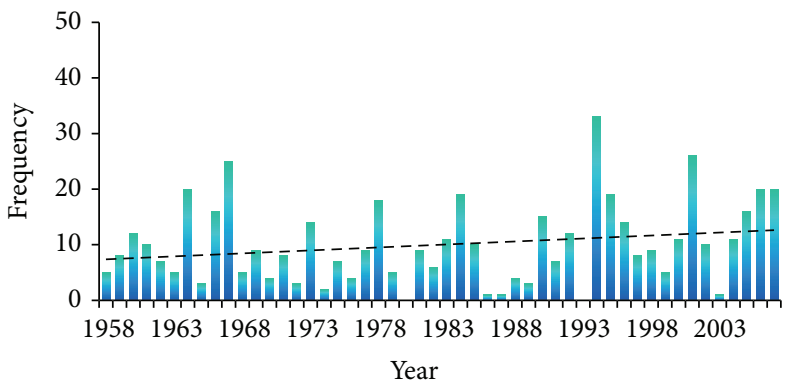

(c) Daegu

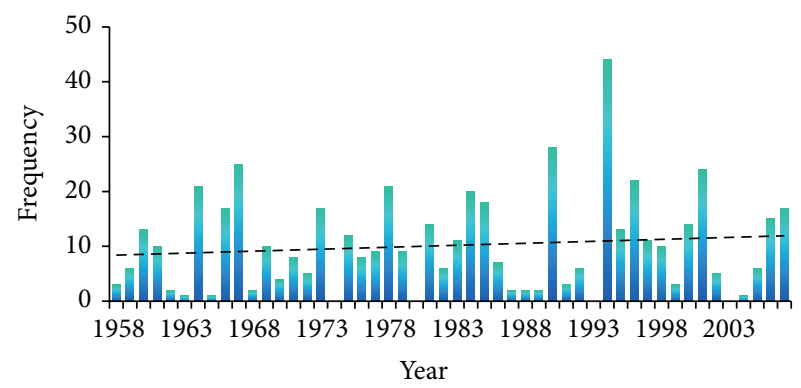

(e) Busan

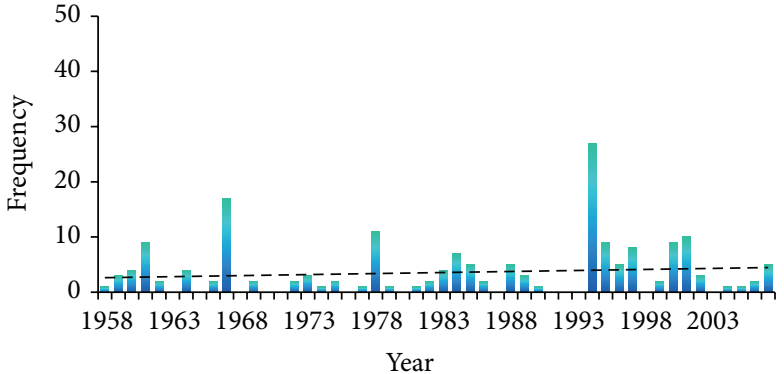

(b) Incheon

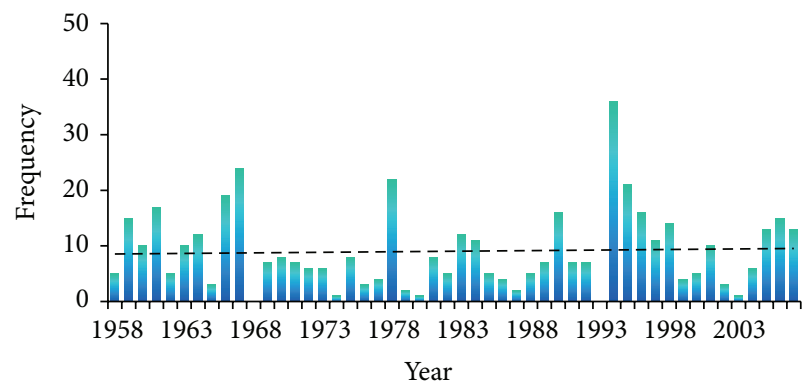

(d) Gwangju

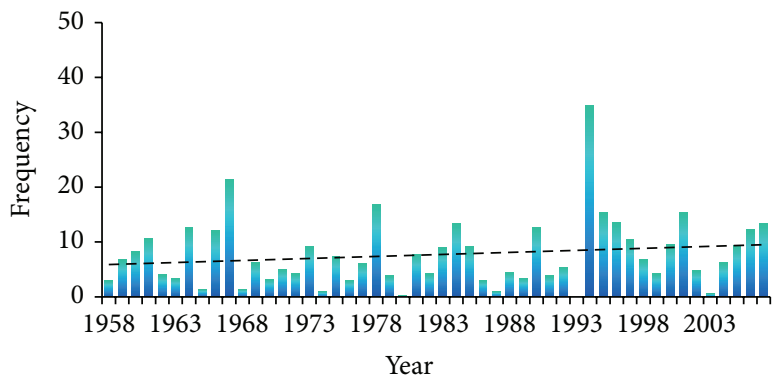

(f) Average

FIGURE 2: Interannual variation in TN days and their linear trends in the five cities in Republic of Korea.

linear trend indicates that the number of TN days in Seoul and Daegu will increase by 13.5 days/100 y and 10.7 days/100 y, respectively. Meanwhile, the number of TN days in Gwangju and Incheon will increase by only 2.0 days/100 $\mathrm{y}$ and 3.7 days $100 \mathrm{y}$, respectively. It shows that the number and trends of TN days are clearly impacted by the urban heat island effect and the geographic location of the cities $[23,24]$. The extraordinarily large number of TN days in 1994, irrespective of geographic location, was related to the record-breaking hot summer in Republic of Korea [36]. We can also see an abrupt increase in the number of TN days after 1993, particularly in Seoul and Daegu. This may be related to the abrupt decadal change in the East Asia summer monsoon in mid to late 1990 [37-39]. Ha and Yun [24] also showed that the number of TN days in Seoul was nearly three times higher during the period 1994-2008, compared with the annual numbers during 19791993. This abnormal event may result in a positive shift in the rate of occurrence of $\mathrm{TN}$ days, which implies that the detection of a change-point in the sequence of data may be affected by this event. Therefore, this effect will be considered in more detail in Section 4.

\section{Bayesian Analysis}

In this section, a Bayesian analysis is presented for the detection of a change-point at an unknown time point in the TN days, which is modeled by an independent Poisson random variable. Some applications of the related topics are studied by Chu and Zhao [15] and Zhao and Chu [40, 41].

Let $\mathbf{y}=\left(y_{1}, y_{2}, \ldots, y_{n}\right)$ be the sample of the TN occurrences over 50 years. Given the observations, we assume that a single change-point model could have occurred at any time point $m$, and consider two types of model, a no-change model and a single change model, to examine whether there is a change in the frequency of TN days. The models used are as follows: 
$\operatorname{model} M_{0}$ :

$$
Y_{i} \sim P_{0}\left(\lambda_{o}\right), \quad i=1,2, \ldots, n ;
$$

$\operatorname{model} M_{1}$ :

$$
\begin{gathered}
Y_{i} \sim P_{0}(\lambda), \quad i=1,2, \ldots, m, \\
Y_{i} \sim P_{0}(\phi), \quad i=m+1, \ldots, n,
\end{gathered}
$$

where $P_{0}(\lambda)$ denotes the Poisson distribution with parameter $\lambda$ and $m$ is an unknown parameter that is called the changepoint of model $M_{1}$. Studies on change-point problems are generally divided into two parts. The first part tests the existence of changes using the Bayes factor or posterior probability, and the second part estimates the parameters of the selected change-point model.

Bayesian model selection or hypothesis testing requires specification of prior distributions for the parameters of the above model in (3). In this paper, informative priors are assumed for the parameters of each model. For models $M_{0}$ and $M_{1}$, prior distributions for $\lambda_{0}$ and $(\lambda, \phi)$ are gamma distributions, as expressed in the following forms, respectively:

$$
\begin{gathered}
\pi\left[\lambda_{0}\right]=\frac{b^{a}}{\Gamma(a)} \lambda_{0}^{a-1} \exp \left(-b \lambda_{0}\right), \\
\pi[\lambda]=\frac{\beta^{\alpha}}{\Gamma(\alpha)} \lambda^{\alpha-1} \exp (-\beta \lambda) \\
\pi[\phi]=\frac{\delta^{\gamma}}{\Gamma(\gamma)} \phi^{\gamma-1} \exp (-\delta \phi),
\end{gathered}
$$

where the gamma function is defined as $\Gamma(x)=\int_{0}^{\infty} t^{x-1} e^{-t} d t$. In addition, the random variable $m$ is assumed to be a discrete uniform distribution.

The posterior probability for each model is useful for selecting one model from more than two models. Given prior distributions, the posterior probability of models $M_{0}$ and $M_{1}$ is obtained by Bayes theorem:

$$
P\left(M_{i} \mid \mathbf{y}\right)=\frac{P\left(\mathbf{y} \mid M_{i}\right) P\left(M_{i}\right)}{\sum_{i=0}^{1} P\left(\mathbf{y} \mid M_{i}\right) P\left(M_{i}\right)}, \quad i=0,1,
$$

where $P\left(M_{i}\right)$ is the prior probability of each model, that is, $P\left(M_{0}\right)=P\left(M_{1}\right)=0.5$, since there is no prior information regarding which one of the models is preferable.

To obtain the posterior probability in (6), we need to specify a marginal distribution of $\mathbf{y}$ for each model. From (3) and (5), the marginal distribution of $\mathbf{y}$ for each model is obtained by, respectively,

$$
\begin{gathered}
P\left(\mathbf{y} \mid M_{0}\right) \propto \frac{b^{a}}{\Gamma(a)} \frac{\Gamma\left(a+\sum_{i=1}^{n} y_{i}\right)}{[n+b]^{a+\sum_{i=1}^{n} y_{i}},} \\
P\left(y \mid M_{1}\right) \propto \frac{\beta^{\alpha} \delta^{\gamma}}{\Gamma(\alpha) \Gamma(\gamma)} \sum_{m=1}^{n-1} \frac{\Gamma\left(\alpha+\sum_{j=1}^{m} y_{j}\right)}{[m+\beta]^{\alpha+\sum_{j=1}^{m} y_{j}}} \\
\cdot \frac{\Gamma\left(\gamma+\sum_{j=m+1}^{n} y_{j}\right)}{[n-m+\delta]^{\gamma+\sum_{j=m+1}^{n} y_{j}}} P(m),
\end{gathered}
$$

TABLE 2: The posterior probabilities for each model.

\begin{tabular}{lcc}
\hline City & \multicolumn{2}{c}{ Posterior probability } \\
\hline Seoul & $P\left(M_{0} \mid y\right)$ & $P\left(M_{1} \mid y\right)$ \\
Incheon & $3.7 * 10^{-11}$ & 1.0 \\
Daegu & 0.00259 & 0.99741 \\
Kwangju & 0.00089 & 0.99911 \\
Busan & 0.77478 & 0.22522 \\
\hline
\end{tabular}

where $P(m)$ is discrete uniform distribution on the set $\{1,2, \ldots, n-1\}$. Using (6) and (8), we can select the most plausible model that has the largest posterior probability.

For each model, the posterior probabilities in (6) and (8) are presented in Table 2. For the selected change-point model $M_{1}$, we consider the Bayes estimation of the parameters. First, for fixed $m$, the conditional joint posterior distribution is

$$
\begin{aligned}
& \pi[\lambda, \phi \mid y, m] \\
& \quad \propto \lambda^{t_{1}} \phi^{t_{2}} \exp (-(m+\beta) \lambda) \exp (-(n-m+\delta) \phi),
\end{aligned}
$$

where $t_{1}=\alpha+\sum_{i=1}^{m} y_{i}$ and $t_{2}=\gamma+\sum_{i=m+1}^{n} y_{i}$.

The probability distribution function of the data $y$ that are only conditional on the change-point $m, P(y \mid m)$, is proportional to

$$
P(y \mid m) \propto \frac{\Gamma\left(t_{1}\right) \Gamma\left(t_{2}\right)}{[m+\beta]^{t_{1}}[n-m+\delta]^{t_{2}}} .
$$

By integrating the appropriate parameters in (9), the conditional posterior distributions $\pi(\lambda \mid m, y)$ and $\pi(\phi \mid m, y)$, denoting before and after the change-point, are obtained by the following forms, respectively:

$$
\begin{gathered}
\pi(\lambda \mid m, y)=G\left(t_{1}, m+\beta\right), \\
\pi(\phi \mid m, y)=G\left(t_{2}, n-m+\delta\right),
\end{gathered}
$$

where $G(c, d)$ denotes the gamma distribution with parameters $c$ and $d$.

Since $m$ is unknown, the marginal posterior distributions $\pi(m \mid y)$ are derived using the Bayes theorem in (10). The marginal density of the change-point $m=1,2, \ldots, n-1$ is calculated by

$$
\begin{aligned}
\pi(m \mid y) & =\frac{P(y \mid m) P(m)}{\sum_{m=1}^{n-1} P(y \mid m) P(m)} \\
& \propto \frac{\Gamma\left(t_{1}\right) \Gamma\left(t_{2}\right)}{[m+\beta]^{t_{1}}[n-m+\delta]^{t_{2}}} P(m) .
\end{aligned}
$$

This discrete distribution gives, for each time point, the posterior probability of a shift in the intensity rate, assuming a change occurred with uncertainty. Finally, the marginal posterior distributions $\pi(\lambda \mid y)$ and $\pi(\phi \mid y)$ are obtained 
by averaging the corresponding conditional posterior density with respect to the posterior mass function of $m$ :

$$
\begin{aligned}
& \pi(\lambda \mid y)=\sum_{m=1}^{n-1} \pi(\lambda \mid m, y) \pi(m \mid y) \\
& \pi(\phi \mid y)=\sum_{m=1}^{n-1} \pi(\phi \mid m, y) \pi(m \mid y) .
\end{aligned}
$$

Note that the marginal distributions of $\lambda$ and $\phi$ appear as finite mixtures of gamma distributions weighted by the $n-1$ values of the marginal posterior density of the change-point $m$.

\section{Results and Discussions}

To choose one model from the two models, $M_{0}$ and $M_{1}$, we computed the posterior probability in (6) for the TN days. The results were obtained by using MATHEMATICA [42] because of practical limitation of using gamma functions. To obtain the posterior probability in (6), the exact value of gamma function must be calculated. That is, if any real value of the gamma function in (10), $t_{1}$ ( or $t_{2}$ ), is greater than a prespecified value, then the gamma function in most of programs returns a value of infinity and has a value out of range. Therefore, it is hard to calculate the quantity of the posterior probability. However, MATHEMATICA [42] can compute an exact value of gamma function for any real value and it provides the exact posterior probability. In our simulation, to consider the noninformative prior, we assumed that only small amount of prior information is available and assign small values to $a=b=\alpha=\beta=\delta=\gamma=0.5$.

First, we conducted the sequence version of MannKendall test [43] to detect the existence of any step changepoints in our data. The sequential version of Mann-Kendall test enables detection of the approximation time of occurrence of trend by locating the intersection of the forward statistic and backward statistic. The forward sequential statistic is estimated using the original time series data, from the first to last data point. The value of backward sequential statistic is estimated using the reversed time series data, starting from the end of the time series. The result of sequential Mann-Kendall test is presented in Figure 3.

In case of Seoul, an apparently positive trend from 1973 to 2007 is observed where the change points have been also detected in 1994 and 1996 and the change point of Daegu has been detected almost at the 1990s in consideration but it does not indicate any recognizable trend. The sequential MannKendall plot for Gwangju displays two points of intersection at 1976 and 1997, respectively, though they are not significant at chosen level of significance. The forward and backward plots for Incheon and Busan intersect each other for several times and there is not a meaningful trend or a sudden change in the time series.

The posterior probabilities for each model under the assumption of equal prior model probability are presented in Table 2. For Seoul, Incheon, and Daegu, the posterior probability of model $M_{1}$ is greater than that of $M_{0}$; therefore, these select the change-point model $M_{1}$. However, Kwangju
TABLE 3: Bayes estimates for parameters $\lambda$ and $\phi$.

\begin{tabular}{lcc}
\hline City & \multicolumn{3}{c}{ Parameters } \\
& $\lambda$ & $\phi$ \\
\hline Seoul & 3.87704 & 10.1015 \\
Incheon & 2.62182 & 5.66166 \\
Daegu & 8.15284 & 13.9978 \\
\hline
\end{tabular}

TABLE 4: Posterior probabilities for each model after omitting the year 1994.

\begin{tabular}{lcc}
\hline City & \multicolumn{2}{c}{ Posterior probability } \\
& $P\left(M_{0} \mid y\right)$ & $P\left(M_{1} \mid y\right)$ \\
\hline Seoul & $5.68 * 10^{-5}$ & 0.9999432 \\
Incheon & 0.808439 & 0.191561 \\
Daegu & 0.276069 & 0.723931 \\
\hline
\end{tabular}

and Busan select a no-change model. This shows that the significant change for the three cities tends to be strong; however, the change tends to be weak for Kwangju and Busan.

Figure 4 shows the posterior probabilities of the changepoint in the TN days of the three cities over Republic of Korea plotted as a function of year. In general, the possibility of change in the given time series is directly proportional to the magnitude of the posterior probability of change. Thus, the maximum posterior probability in the year 1993 indicates that a change in the TN days of three cities occurred in or around 1993.

Under model $M_{1}$, the exact Bayes estimation of the unknown change-point and parameters $\lambda$ and $\phi$ (means before and after the change-point) is derived. Table 3 shows the estimated posterior means of $\lambda$ and $\phi$. For three cities, it is found that there are considerably more TN days after the change-point than those before the change-point. This suggests that TN days' occurrence has become more frequent since 1993.

The marginal posterior density functions of $\lambda$ and $\phi$ for the period before and after change-point year 1993 are presented in Figure 5. The solid line is a marginal posterior density function of TN days before the change $\pi(\lambda \mid y)$, and the dotted line is that of TN days after the change $\pi(\phi \mid y)$. Since the overlap in the tail areas between these two posterior distributions is weak, the distribution of the marginal posterior density function of the change-point also confirms that change in the TN days occurred around 1993.

As mentioned above, it is seen that the extraordinarily large number of TN days occurred in 1994. The sample average for the counts of TN days after 1993 may be higher and the linear trend may reveal an increasing pattern since it is affected by the event. Therefore, this may give a misleading result in a change-point problem. To examine the effect, first, the event is removed and then the change-point detection method is applied as before. The result of the Bayesian analysis on the change-point of annual counts of TN days over three cities is listed in Tables 4 and 5 and Figure 6.

In Table 4 , a single change model $M_{1}$ is selected for Seoul and Daegu because it is associated with the largest probability. 

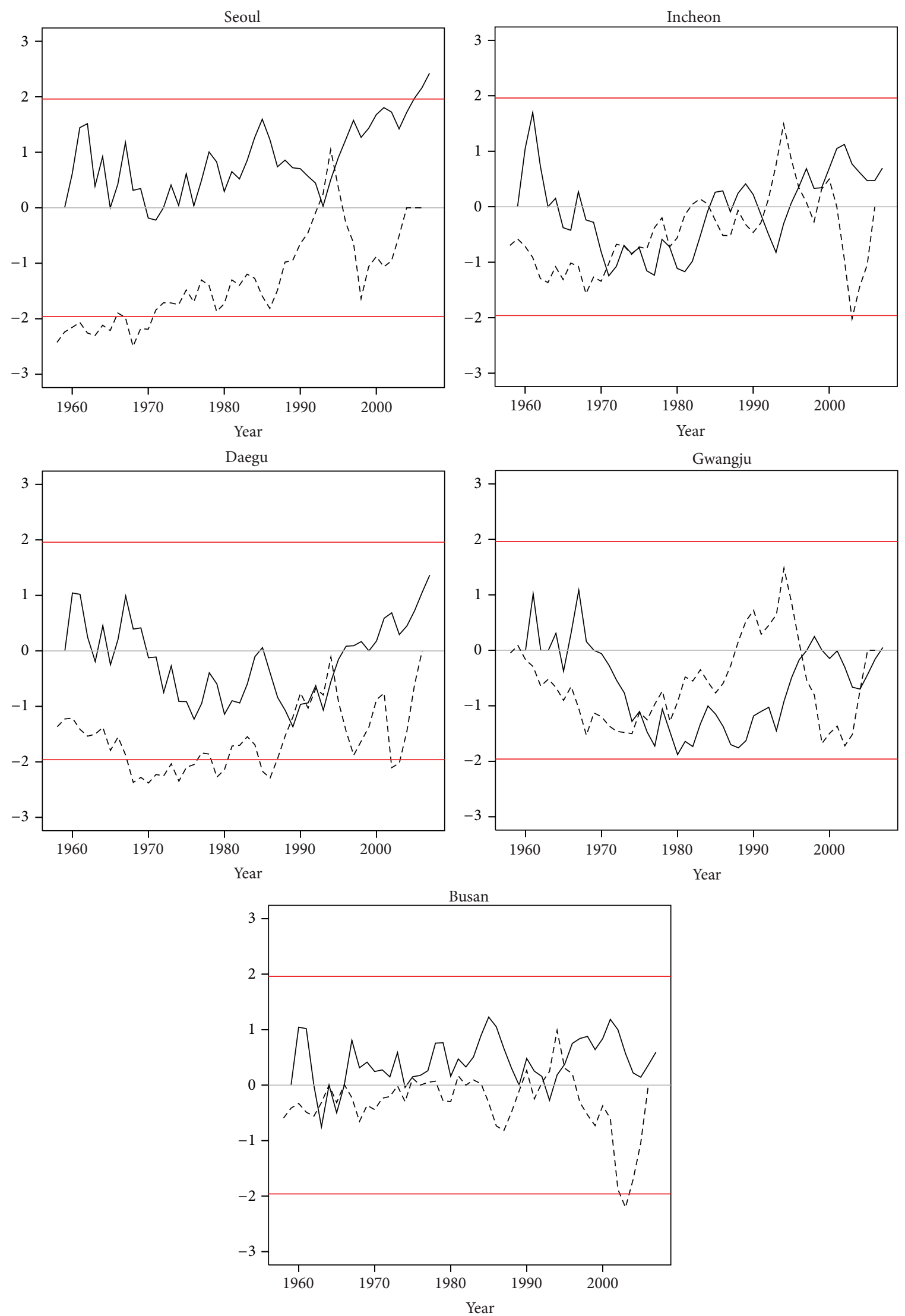

Figure 3: Sequential Mann-Kendall statistic values of forward sequence (solid line), backward sequence (dashed line), and confidence limit at $5 \%$ (red line) for time series. 

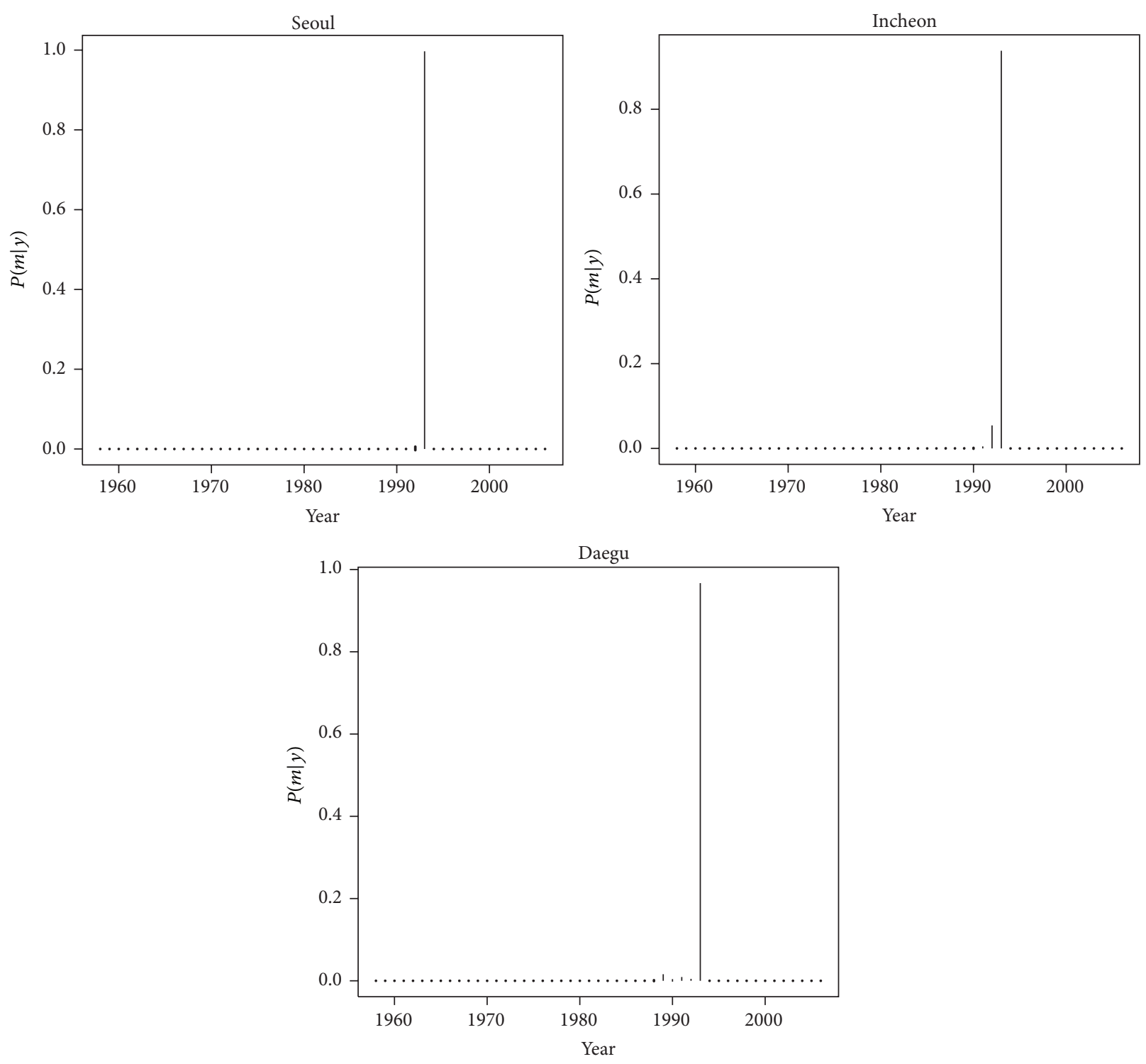

FIGURE 4: Posterior probability for the change-point, $P(m \mid y)$.

TABLE 5: Bayes estimates for the parameters, $\lambda$ and $\phi$ after omitting the year 1994 .

\begin{tabular}{lcc}
\hline City & \multicolumn{3}{c}{ Parameters } \\
& $\lambda$ & \multicolumn{1}{c}{$\phi$} \\
\hline Seoul & 3.87939 & 8.32093 \\
Incheon & 2.71047 & 3.13115 \\
Daegu & 8.35886 & 13.2678 \\
\hline
\end{tabular}

This is consistent with previous results and does not depend on the outlier event. However, in the case of Incheon a nochange model is preferred after removing the event. This is different to the previous result, which is influenced by the outlier event.

In Figure 6, the maximum posterior probability of the change-point occurred in 1993, which implies a rate increase after the year 1993. This result coincides with the previous result.

For Seoul and Daegu in Table 5, it is seen that the number of TN days after the change-point is considerably larger than that before the change-point. The higher average of the Poisson mean rate after the change confirms the direction of change, that is, an increased activity of TN days. However, there is little difference in the number of TN days before and after the change-point for Incheon.

In Figure 6, the marginal posterior densities for the mean rates of Poisson rates of the TN occurrences before and after the change-point show a slight overlap, implying a significant rate increase after the change even though the year with the highest count of TN days was removed.

For Seoul and Daegu, the result of the Bayesian changepoint analysis suggests that a single change occurred around 1993 and it is not influenced by this outlier event among the 

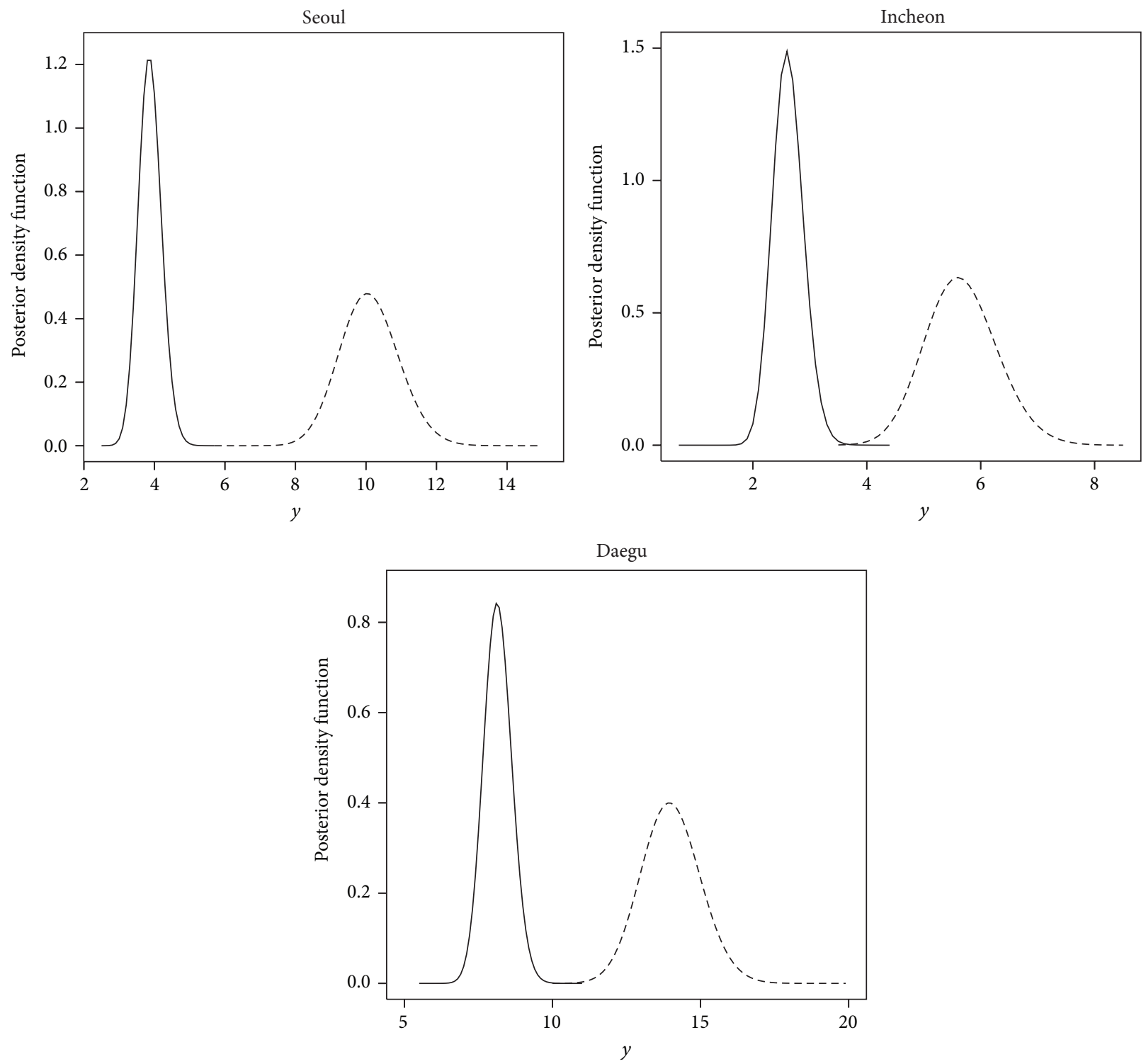

FIGURE 5: Marginal posterior density functions for parameters $\lambda$ and $\phi$ before and after the change-point in 1993.

TN days' counts. However, Incheon shows conflicting results since these depend on this event to detect the change-point. Based on the results, there is inadequate evidence in favor of increased activity of TN days in Incheon.

In the comparison of averaged $\mathrm{TN}$ days after regime shift year, the averaged TN days in Seoul and Daegu increase significantly, by more than $150 \%$, after the change-point year (1993), even when the extraordinary year, 1994, is excluded. Ha and Yun [24] also showed that the annual number of TN days in Seoul was nearly three times larger during the period 1994-2008 compared with during the period 19791993. However, the annual number of TN days in Gwangju and Busan only increased by less than $130 \%$ when the TN days in 1994 are excluded. It confirms that a change-point in the frequency of TN days only occurs for two cities, Seoul and Daegu.

\section{Conclusions}

It is well known that the frequency and intensity of temperature extremes are influenced by the combined effects of global warming and localized forcing, such as urbanization. The increase in daily minimum temperature is more significant than that of the maximum temperature. Therefore, the frequency and intensity of tropical night (TN) days or warm night days have significantly increased due to enhanced warming and rapid urbanization in Republic of Korea. In this study, the existence of a change-point in the time series of TN days in the five major cities (Seoul, Busan, Daegu, Incheon, and Gwangju) over Republic of Korea was investigated using Bayesian change-point analysis during a 50-year period (1958-2007). A Bayesian analysis was performed for the detection of a change-point at an unknown time point in 

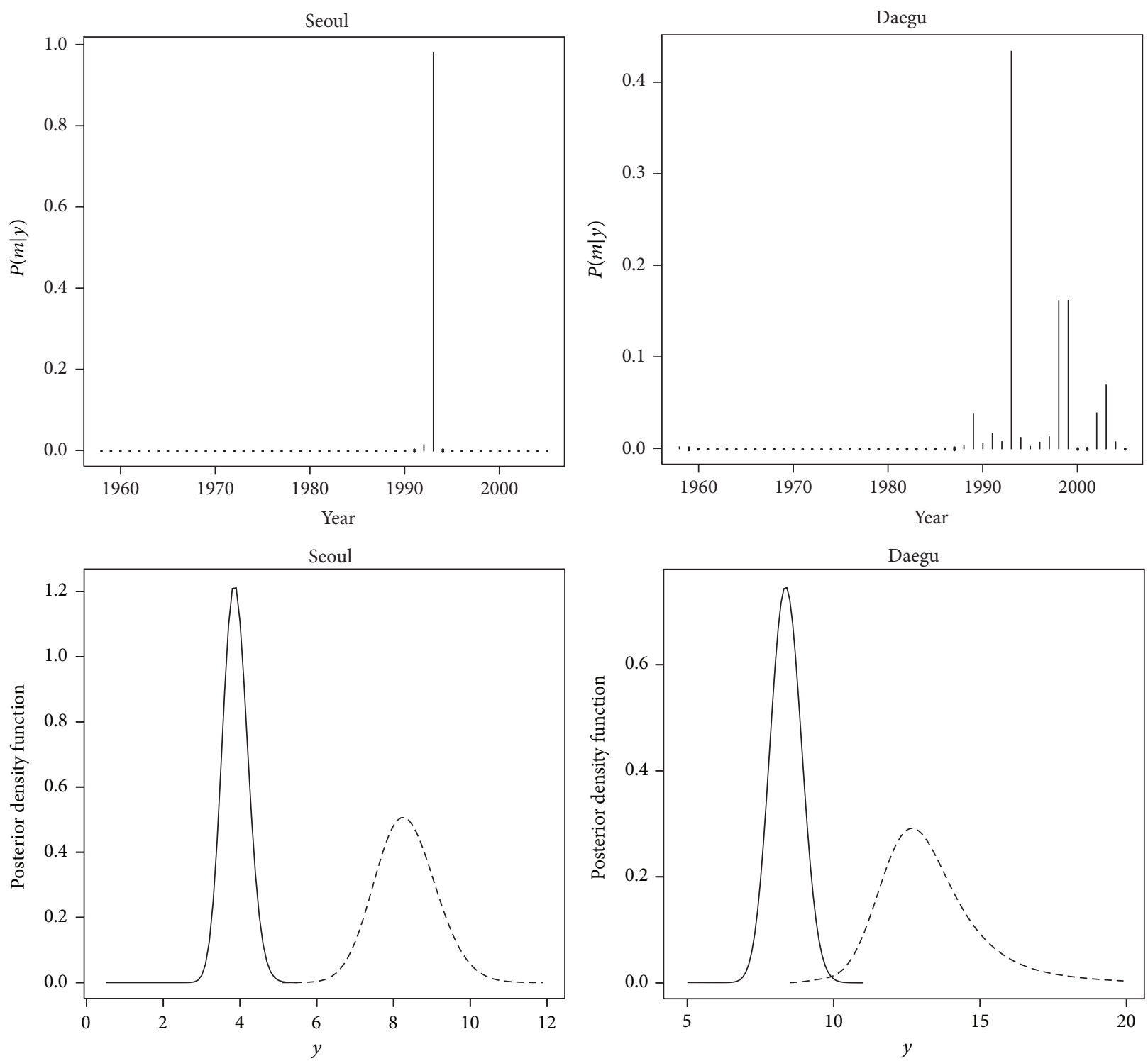

FIGURE 6: Posterior probability for the change-point, $P(m \mid y)$, and the marginal posterior density functions for $\lambda$ and $\phi$ before and after the change-point after omitting the year 1994.

the TN days, which is modeled by an independent Poisson random variable.

The distribution of the marginal posterior density function of the change-point confirms that change in the TN day frequency occurred around 1993 in three cities, Seoul, Daegu, and Incheon. However, when we excluded the extraordinary year, 1994, the Bayesian change-point analysis suggests that a single change occurred around 1993 only in Seoul and Daegu, but it prefers a no-change model in Incheon. The averaged TN days before and after the change-point year (1993) also showed that a change in the TN days occurred around 1993 in only two cities, Seoul and Daegu. The averaged TN days in Seoul and Daegu significantly increased, by more than $150 \%$, after the change-point year (1993), even when the extraordinary year, 1994, is omitted. The abrupt increase of TN days around 1993/1994 in the two cities over Republic of Korea might be related to the significant decadal change in the East Asian summer monsoon that occurred around the mid 1990s and rapid urbanization in the selected cities $[24,37,39]$. This result suggests that the change in TN day frequency is modulated not only by large scale forcing (e.g., global warming, circulation change) but also by the local environment of the city (e.g., geographic location, city size).

In this study, we only investigated the existence of changepoint in the time series of TN days. Therefore, more work is needed to investigate the thermodynamical mechanisms of abrupt changes in TN day frequency, including urbanization and large scale circulation changes.

\section{Conflict of Interests}

The authors declare that there is no conflict of interests regarding the publication of this paper. 


\section{Acknowledgment}

This work was funded by the Korea Meteorological Administration Research and Development Program under Grant CATER 2012-3081.

\section{References}

[1] G. R. McGregor, C. A. T. Ferro, and D. B. Stephenson, "Projected changes in extreme weather and climate events in Europe," in Extreme Weather Events and Public Health Responses, Springer, New York, NY, USA, 2005.

[2] J. Y. Kim, D. G. Lee, I. S. Park, B. C. Choi, and J. S. Kim, "Influences of heat waves on daily mortality in South Korea," Atmosphere, vol. 16, no. 12, pp. 269-278, 2006.

[3] J. Kyselý, R. Huth, and J. Kim, "Evaluating heat-related mortality in Korea by objective classifications of 'air masses," International Journal of Climatology, vol. 30, no. 10, pp. 1484-1501, 2010.

[4] D. R. Easterling, G. A. Meehl, C. Parmesan, S. A. Changnon, T. R. Karl, and L. O. Mearns, "Climate extremes: observations, modeling, and impacts," Science, vol. 289, no. 5487, pp. 20682074, 2000.

[5] M. Haylock and N. Nicholls, "Trends in extreme rainfall indices for an updated high quality data set for Australia, 1910-1998," International Journal of Climatology, vol. 20, no. 13, pp. 15331541, 2000.

[6] C. Schär, P. L. Vidale, D. Lüthi et al., "The role of increasing temperature variability in European summer heatwaves," Nature, vol. 427, no. 6972, pp. 332-336, 2004.

[7] P. Zhai, X. Zhang, H. Wan, and X. Pan, "Trends in total precipitation and frequency of daily precipitation extremes over China," Journal of Climate, vol. 18, no. 7, pp. 1096-1108, 2005.

[8] S.-K. Min and A. Hense, "A Bayesian assessment of climate change using multimodel ensembles. Part I: global mean surface temperature," Journal of Climate, vol. 19, no. 13, pp. 3237-3256, 2006.

[9] S.-K. Min and A. Hense, "A Bayesian assessment of climate change using multimodel ensembles. Part II. Regional and seasonal mean surface temperatures," Journal of Climate, vol. 20, no. 12, pp. 2769-2790, 2007.

[10] L. A. Vincent and É. Mekis, "Changes in daily and extreme temperature and precipitation indices for Canada over the twentieth century," Atmosphere-Ocean, vol. 44, no. 2, pp. 177-193, 2006.

[11] T. Ding, W. Qian, and Z. Yan, "Changes in hot days and heat waves in China during 1961-2007,' International Journal of Climatology, vol. 30, no. 10, pp. 1452-1462, 2010.

[12] F. G. Kuglitsch, A. Toreti, E. Xoplaki et al., "Heat wave changes in the eastern Medtierranean since 1960," Geophysical Research letters, vol. 37, no. 4, Article ID L04082, 2010.

[13] J. Kyselý, "Recent severe heat waves in central Europe: how to view them in a long-term prospect?" International Journal of Climatology, vol. 30, no. 1, pp. 89-109, 2010.

[14] J. J. Egozcue and C. Ramis, "Bayesian hazard analysis of heavy precipitation in Eastern Spain," International Journal of Climatology, vol. 21, no. 10, pp. 1263-1279, 2001.

[15] P.-S. Chu and X. Zhao, "Bayesian change-point analysis of tropical cyclone activity: the central North Pacific case," Journal of Climate, vol. 17, no. 24, pp. 4893-4901, 2004.

[16] C. Tebaldi, L. O. Mearns, D. Nychka, and R. L. Smith, "Regional probabilities of precipitation change: a Bayesian analysis of multimodel simulations," Geophysical Research Letters, vol. 31, no. $24,2004$.

[17] M. N. Khaliq, T. B. M. J. Ouarda, A. St.-Hilaire, and P. Gachon, "Bayesian change-point analysis of heat spell occurrences in Montreal, Canada," International Journal of Climatology, vol. 27, no. 6, pp. 805-818, 2007.

[18] IPCC, "Climate change 2007: the physical science basis," in Contribution of Working Group I to the Fourth Assessment Report of the Intergovernmental Panel on Climate Change, D. L. Albritton and L. G. MeiraFilho, Eds., Cambridge University Press, Cambridge, UK, 2007.

[19] S. A. Changnon, K. E. Kunkel, and B. C. Reinke, "Impacts and responses to the 1995 heat wave: a call to action," Bulletin of the American Meteorological Society, vol. 77, no. 7, pp. 1497-1506, 1996.

[20] A. M. G. K. Tank and G. P. Können, "Trends in indices of daily temperature and precipitation extremes in Europe, 1946-99," Journal of Climate, vol. 16, no. 22, pp. 3665-3680, 2003.

[21] D. R. Easterling, B. Horton, P. D. Jones et al., "Maximum and minimum temperature trends for the globe," Science, vol. 277, no. 5324, pp. 364-367, 1997.

[22] Y.-H. Kim and J.-J. Baik, "Daily maximum urban heat island intensity in large cities of Korea," Theoretical and Applied Climatology, vol. 79, no. 3-4, pp. 151-164, 2004.

[23] L. V. Alexander, X. Zhang, T. C. Peterson et al., "Global observed changes in daily climate extremes of temperature and precipitation," Journal of Geophysical Research D: Atmospheres, vol. 111, no. 5, Article ID D05109, 2006.

[24] K.-J. Ha and K.-S. Yun, "Climate change effects on tropical night days in Seoul, Korea," Theoretical and Applied Climatology, vol. 109, no. 1-2, pp. 191-203, 2012.

[25] H.-S. Jung, Y. Choi, J.-H. Oh, and G.-H. Lim, "Recent trends in temperature and precipitation over South Korea," International Journal of Climatology, vol. 22, no. 11, pp. 1327-1337, 2002.

[26] C.-H. Ho, J.-Y. Lee, M.-H. Ahn, and H.-S. Lee, "A sudden change in summer rainfall characteristics in Korea during the late 1970s," International Journal of Climatology, vol. 23, no. 1, pp. 117-128, 2003.

[27] G. Y. Choi, W. T. Kwon, K. O. Boo, and Y. M. Cha, "Recent spatial and temporal changes in means and extreme events of temperature and precipitation across the Republic of Korea," Journal of the Korean Geographical Society, vol. 43, no. 5, pp. 681-700, 2008.

[28] C. Kim and M. S. Suh, "Change-point in the recent (1976-2005) precipitation over South Korea," Atmosphere, vol. 18, no. 2, pp. 111-120, 2008 (Korean).

[29] C. Kim, M.-S. Suh, and K.-O. Hong, "Bayesian changepoint analysis of the annual maximum of daily and subdaily precipitation over South Korea," Journal of Climate, vol. 22, no. 24, pp. 6741-6757, 2009.

[30] Y. E. Choi, "Trends on temperature and precipitation extreme events in Korea ," Journal of the Korean Geographical Society, vol. 39, no. 5, pp. 711-721, 2004.

[31] G. Y. Choi and W. T. Kwon, "Spatial-temporal patterns and recent changes of tropical night phenomenon in South Korea," Journal of the Korean Geographical Society, vol. 40, no. 6, pp. 730-747, 2005.

[32] J. Y. Kim and S. H. Lee, "The distribution of heat waves and its cause in South Korea," Journal of the Korean Geographical Society, vol. 42, no. 3, pp. 332-343, 2007. 
[33] J. Kyselý and J. Kim, "Mortality during heat waves in South Korea, 1991 to 2005: how exceptional was the 1994 heat wave?" Climate Research, vol. 38, no. 2, pp. 105-116, 2009.

[34] L. Perreault, J. Bernier, B. Bobée, and E. Parent, "Bayesian change-point analysis in hydrometeorological time series. Part 1. The normal model revisited," Journal of Hydrology, vol. 235, no. 3-4, pp. 221-241, 2000.

[35] L. Perreault, J. Bernier, B. Bobée, and E. Parent, "Bayesian change-point analysis in hydrometeorological time series. Part 2. Comparison of change-point models and forecasting," Journal of Hydrology, vol. 235, no. 3-4, pp. 242-263, 2000.

[36] S.-J. Heo, K.-J. Ha, and S.-E. Moon, "Characteristic features of the East Asian summer monsoon during 1993 and 1994," Journal of the Korean Meteorological Society, vol. 33, no. 4, pp. 737-751, 1997 (Korean).

[37] M. Kwon, J.-G. Jhun, and K.-J. Ha, "Decadal change in east Asian summer monsoon circulation in the mid-1990s," Geophysical Research Letters, vol. 34, no. 21, Article ID L21706, 2007.

[38] W. Kim, J.-G. Jhun, K.-J. Ha, and M. Kimoto, "Decadal changes in climatological intraseasonal fluctuation of subseasonal evolution of summer precipitation over the Korean Peninsula in the mid-1990s," Advances in Atmospheric Sciences, vol. 28, no. 3, pp. 591-600, 2011.

[39] Y. Chikamoto, M. Kimoto, M. Ishii et al., "Predictability of a stepwise shift in Pacific climate during the late 1990s in hindcast experiments using MIROC," Journal of the Meteorological Society of Japan, vol. 90A, pp. 1-21, 2012.

[40] X. Zhao and P.-S. Chu, "Bayesian multiple changepoint analysis of hurricane activity in the eastern North Pacific: a Markov chain Monte Carlo approach," Journal of Climate, vol. 19, no. 4, pp. 564-578, 2006.

[41] X. Zhao and P.-S. Chu, "Bayesian changepoint analysis for extreme events (typhoons, heavy rainfall, and heat waves): an RJMCMC approach," Journal of Climate, vol. 23, no. 5, pp. 10341046, 2010.

[42] Wolfram Research, Mathematics Version 6.0, Wolfram Research, Champaign, Ill, USA, 2007.

[43] R. Sneyers, "On the statistical analysis of series of observations," Technical Note 143, World Meteorological Organization, Geneva, Switzerland, 1990. 

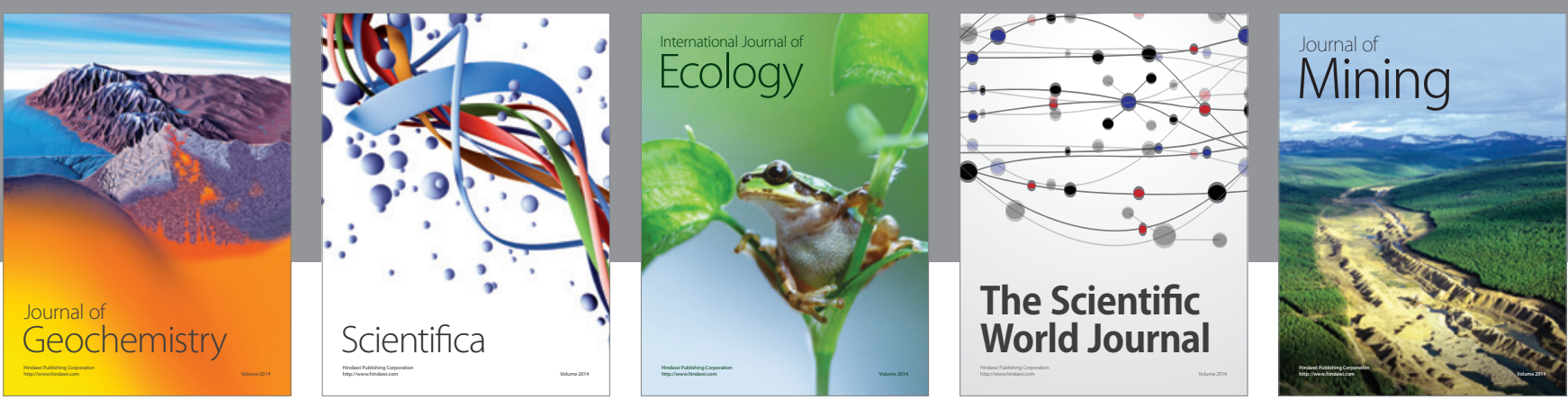

The Scientific World Journal
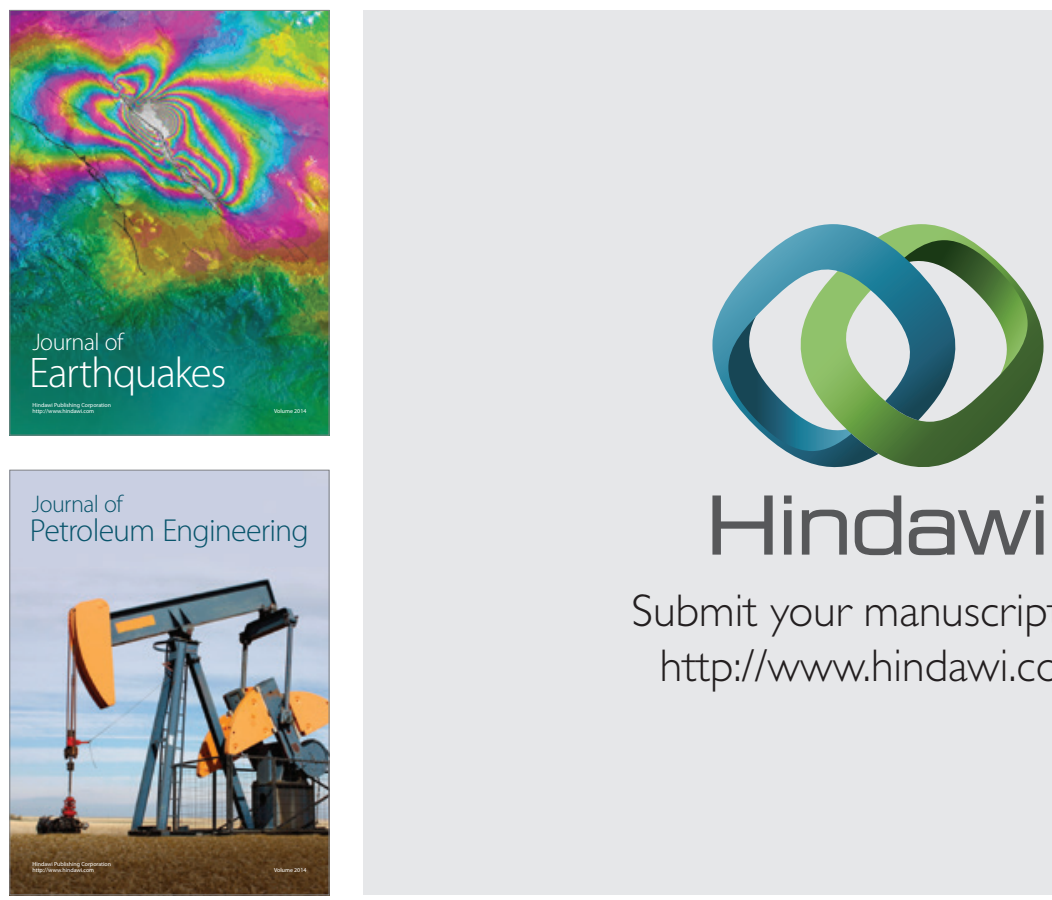

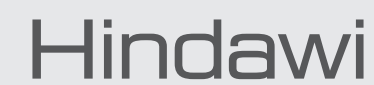

Submit your manuscripts at

http://www.hindawi.com
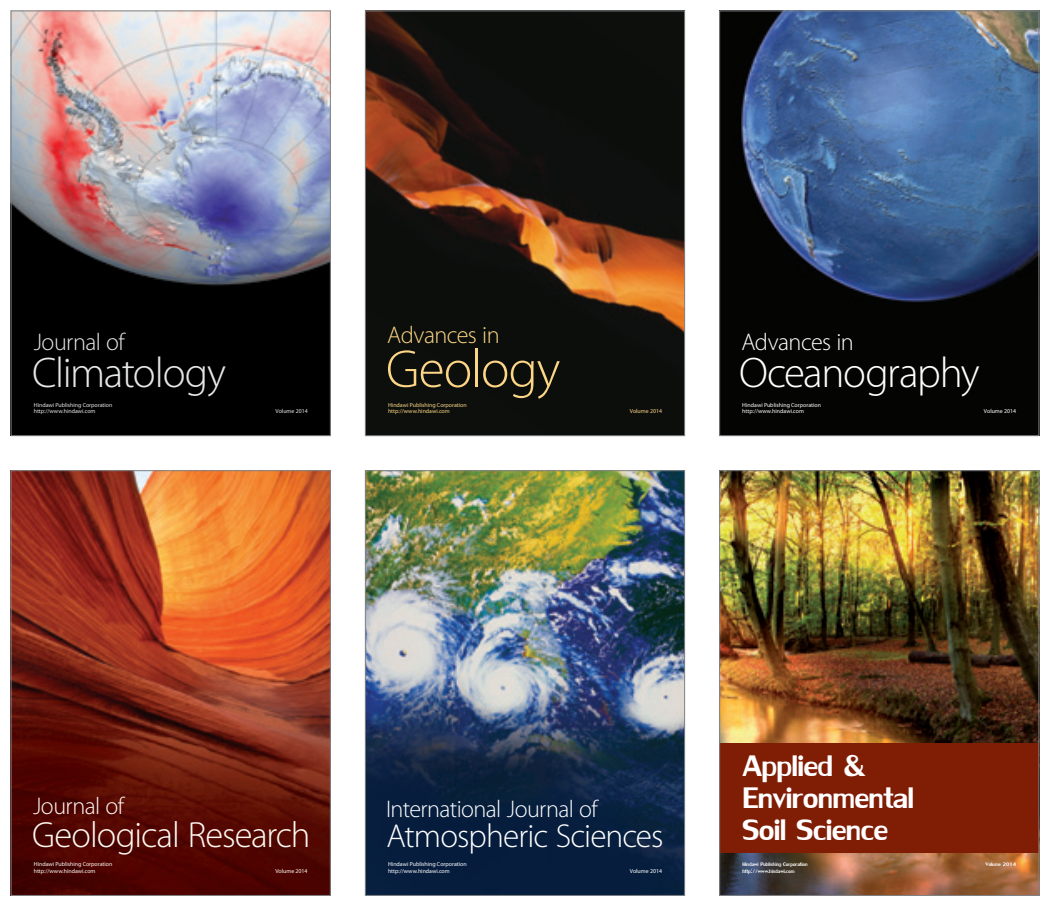
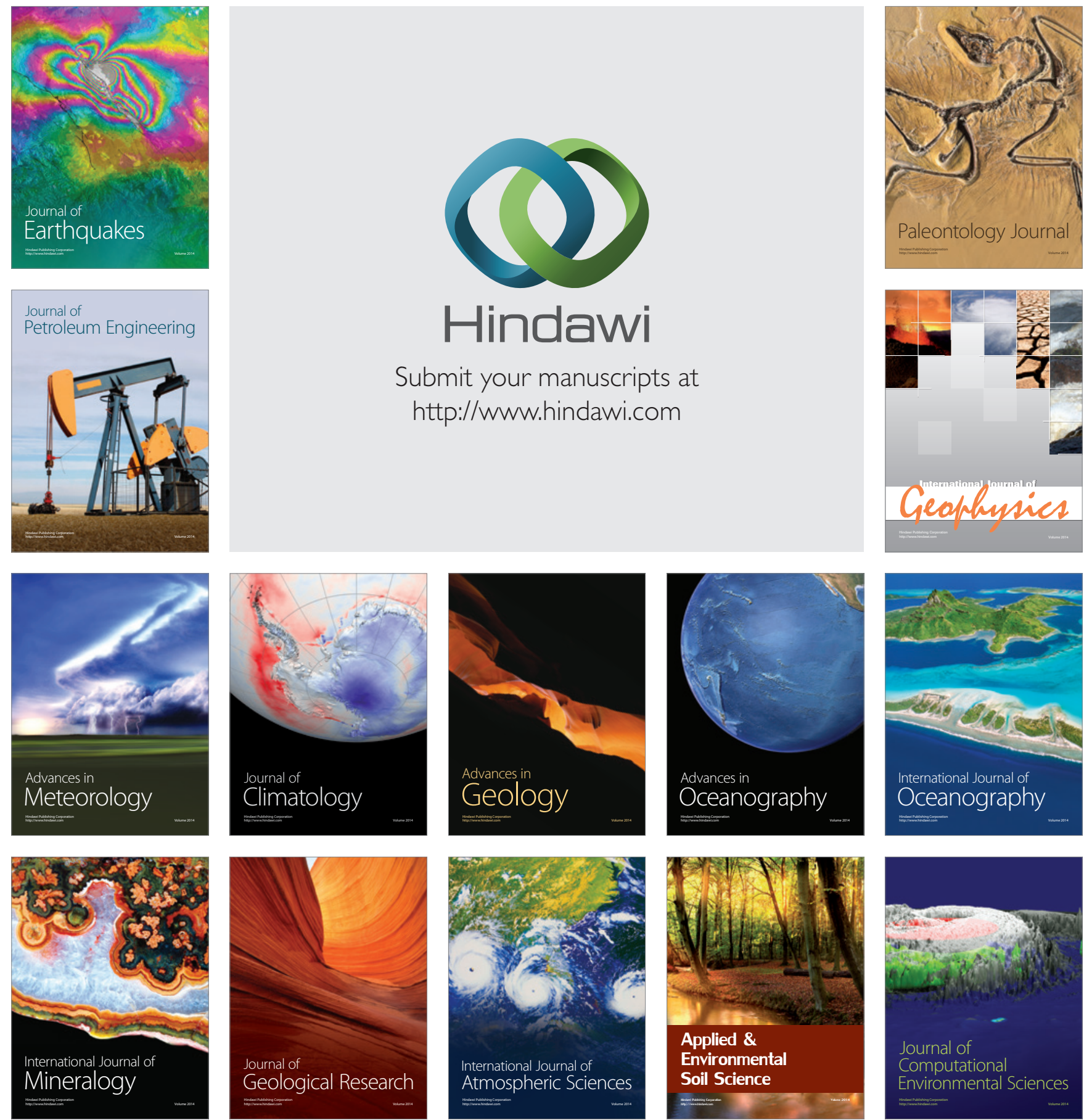